\title{
Explanatory drawings prioritize functional properties at the expense of visual fidelity
}

\author{
Holly Huey $^{\mathrm{a}, 1}$, Xuanchen Lu ${ }^{\mathrm{a}}$, Caren M. Walker ${ }^{\mathrm{a}}$, and Judith E. Fan ${ }^{\mathrm{a}}$

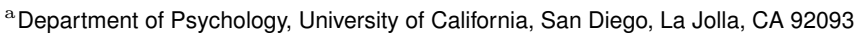 \\ This manuscript was compiled on August 30, 2021
}

\begin{abstract}
Explanatory visualizations play an integral role in communicating mechanistic knowledge - how things work. What characterizes explanatory visualizations, and how do they differ from other modes of visual communication? To explore this question, we prompted people to produce drawings explaining how to operate several simple machines, then conducted a detailed analysis of the semantic information conveyed in each drawing. By comparison with depictive drawings intended only to convey the machine's appearance, we found that explanatory drawings prioritized parts of the machine that move or interact to produce an effect over other parts that were visually salient, but static. Next, we measured how effectively these drawings helped other people extract relevant information about each machine. We found that people were better at inferring which action was needed to operate the machine when they viewed an explanatory drawing than when they viewed a depictive one, but were worse at identifying which machine it represented and which part to interact with. In sum, our findings suggest that the way people spontaneously prioritize functional information when producing visual explanations may be "double-edged," facilitating some aspects of causal inference in downstream learners at the expense of others.
\end{abstract}

natural pedagogy | causal learning | explanation | visual production system, humans are driven to understand how things work and use that knowledge to generate desired outcomes. However, acquiring such mechanistic knowledge firsthand can often be costly in time and effort $(1,2)$ and a large share of the knowledge we have today depends on its faithful transmission from previous generations $(3,4)$. Such prodigious knowledge transmission has been supported by the use of mechanistic explanations, which serve to expose causal links between phenomena, yielding durable and compact mental representations of otherwise fleeting and complex information (5).

What characterizes good mechanistic explanations, and how do they relate to the phenomena they are intended to explain? Prominent theoretical perspectives suggest several hallmark features $(6,7)$, including the decomposition of a system into interacting parts and the specification of causal relations between those parts in the service of some function. Take, for example, a bicycle, which works by transferring power from the movement of the pedals to the drive wheel via a roller chain, propelling it forward. Such an explanation can be contrasted with a merely descriptive report (e.g., "A bicycle has two wheels, pedals, and a chain."), which does not require causal relations to be specified (8), or with a teleological explanation (e.g., "A bicycle is for riding."), which does not require mentioning parts or how they interact (9). In addition to playing a key role in scientific theories (10), there is growing evidence that mechanistic explanations are also privileged in people's intuitive understanding of what constitutes generalizable knowledge about artifacts and biological entities $(11,12)$. The typical strategy in this prior work has been to measure sensitivity to mechanistic information by collecting judgments based on explanations provided by others. As such, it is not yet clear how well these judgments predict what information people actually prioritize when producing explanations, nor how these explanations support key aspects of mechanistic understanding, such as the identification of causally relevant parts and how they interact.

Explanatory visualizations may be especially useful for probing the cognitive processes that enable the communication of mechanistic knowledge (13-16). Visualizations naturally exploit shape-based and spatial cues to both expose the relevant part-based and relational abstractions that underlie mechanistic understanding $(17,18)$, as well as how these abstractions map back onto physical elements of the target system (19-22). Moreover, there is ample evidence that visualizations can facilitate learning and inference by comparison with text alone $(17,23-25)$. Yet while scientific explanations generally make substantial use of graphs, diagrams, and other illustrations $(26,27)$, most previous studies of explanation behavior have focused on verbal explanations (28-31). Thus there is both an opportunity to fill a basic gap in our understanding of how visual explanations work, and leverage their distinctive potential to provide detailed insight into how explanatory abstractions are grounded in our direct experience with causal systems.

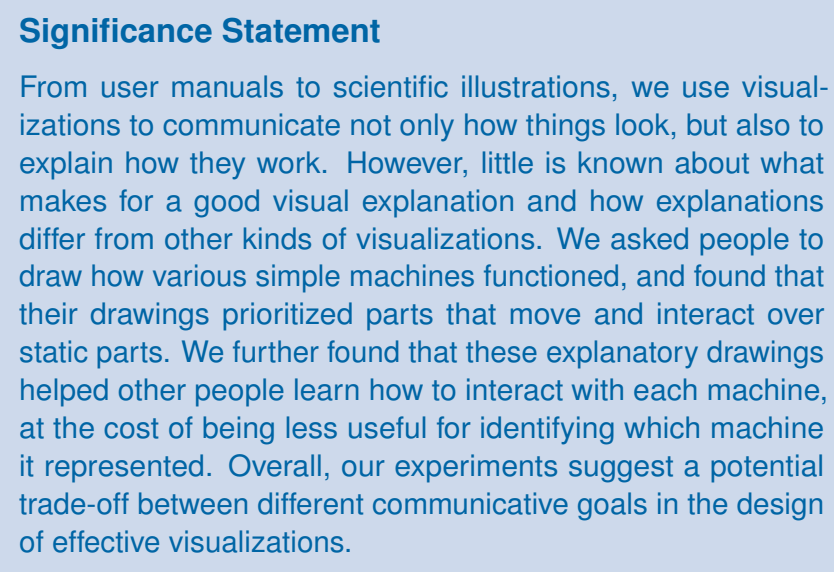

Author contributions: H.H., C.M.W., and J.E.F. designed experiments; H.H. and J.E.F. conducted experiments; H.H., X.L., and J.E.F. analyzed data; and H.H., C.M.W., and J.E.F. interpreted findings and wrote the paper.

The authors declare no conflict of interest.

${ }^{1}$ To whom correspondence should be addressed. E-mail: jefan@ucsd.edu 
Here we investigate the production and use of visual explanations of novel mechanical objects. In particular, we ask how people combine information about structural organization (i.e., what the parts are and how they are arranged) with information about functional organization (i.e., how these parts move and interact) when producing visual explanations, and how viewers map such information from visual explanations back to the target object. One possibility is that functional and structural information are dissociable in the visual explanations people generate. Under this account, these two types of information may trade off with one another, such that drawings prioritizing functional information (e.g., how one gear interacts with another) can be less effective in communicating structural information (e.g., the size and location of each gear). Alternatively, functional and structural information may generally be bound together in visual explanations. Under this account, drawings that are better able to convey functional information would also be predicted to better communicate structural information.

To disentangle these possibilities, we conducted two experimental studies: In Study 1, we ask what information people prioritize when generating visual explanations of novel mechanical objects, as opposed to depictive illustrations. In Study 2 , we examine how these visual explanations guide inferences made by naive observers relying only upon these explanations to learn about these objects. Overall, we found that explanatory drawings emphasized causally relevant information and more effectively communicated which action was needed when interacting with the machine. On the other hand, they were less informative about which machine it corresponded to, or which part to interact with. Together, these findings suggest that people engaging in visual explanation spontaneously prioritize functional information at the expense of visual fidelity, making it easier to understand how causally important parts interact, but not necessarily what they look like or where they are in relation to other parts.

\section{Results}

Study 1A: Eliciting visual explanations and depictions. Our first goal was to identify the semantic properties that characterize visual explanations. To accomplish this goal, we designed six novel machines, composed of simple mechanical elements (i.e., gears, levers, pulleys), and recorded 30s videos demonstrating how to operate each machine. Half of the mechanical elements in each machine were causal, meaning they could be used to produce a desired effect (i.e., turn on a light); the other half of mechanical elements were non-causal. To evaluate the effectiveness of these video demonstrations, we recruited one group of participants $(\mathrm{N}=124)$ to view each video, then identify which mechanical element they should intervene upon to turn on the light. We found that participants could generally do so $(73.7 \%$ correct; $95 \%$ CI: [0.695, 0.778]), affirming that the video demonstrations were sufficiently clear.

We then recruited a second group of participants $(\mathrm{N}=52)$, who produced a drawing of each machine after viewing each video. on three of these trials, participants were prompted to produce a visual explanation to help a naive viewer learn how the machine worked; on the remaining three trials, they were prompted to produce a visual depiction that would help a naive viewer identify which machine it represented (Fig. 1). The resulting dataset contained 300 drawings from 50 unique

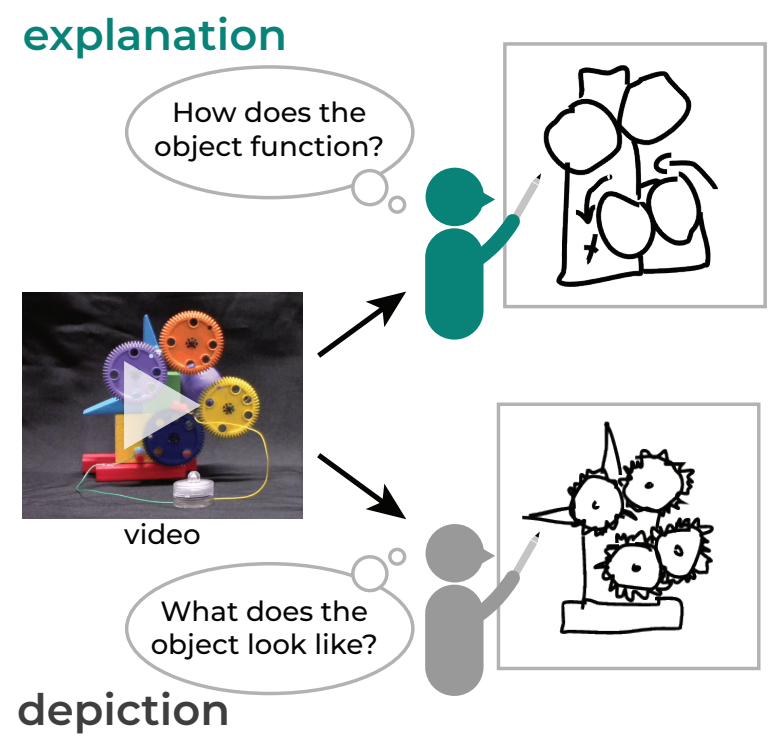

Fig. 1. Study 1: Visual Production Task. On each trial, participants viewed a 30 -second video demonstrating how to operate a machine to turn on a light bulb. After the video finished playing, on half of trials participants were then prompted to produce an explanatory drawing. On the other half of trials, they were prompted to produce a depictive drawing.

participants: 150 visual explanations and 150 depictions. We found that participants used a similar number of strokes (explanation: 20.33; depiction: $18.9 ; b=1.44, t=1.04, p=$ 0.301; Fig. 1B, left) and amount of time drawing in both conditions (explanation: 59300ms; depiction: $57689 \mathrm{~ms} ; b=$ $1144.75, t=0.359, p=0.72$; Fig. $1 \mathrm{~B}$, right), suggesting that they had invested a similar degree of effort when producing both types of drawings. Moreover, they indicate that these simple effort-based measures are insufficient to capture differences in the semantic information conveyed by each type of drawing.

Study 1B: Characterizing semantic content in visual explanations and depictions. To characterize the semantic content contained in these drawings, we crowdsourced annotations from a separate group of naive participants $(\mathrm{N}=280)$. For each stroke in every drawing, we obtained labels from at least three annotators indicating which part of the machine it corresponded to (e.g., "gear", "lever", "structural"). Each of these labels were then further grouped into higher-level semantic categories: causal strokes representing mechanical elements that were causally related to turning on the light bulb, noncausal strokes representing mechanical elements that were not causally related to turning on the light bulb, structural strokes representing structural elements, and symbolic strokes, including arrows and other marks indicating motion and interactions between elements.

Based on these annotations, we discovered that among strokes representing a mechanical element (i.e., gear, lever, or pulley), a greater proportion were devoted to representing causal parts in visual explanations than in depictions (explanation: $58.0 \%$, depiction: $42.0 \%, b=0.382, z=3.44, p=$ $5.9 e-4$; Fig. 1C). Moreover, visual explanations contained more strokes that represented symbols (explanation: $24.8 \%$, depiction: $1.0 \%, b=2.48, t=1.392, p=1.67 e-1)$ and fewer strokes representing structural elements (explanation: $25.0 \%$, depiction: $45.8 \%, b=-2.77, t=-4.86, p=1.31 e-5)$. 


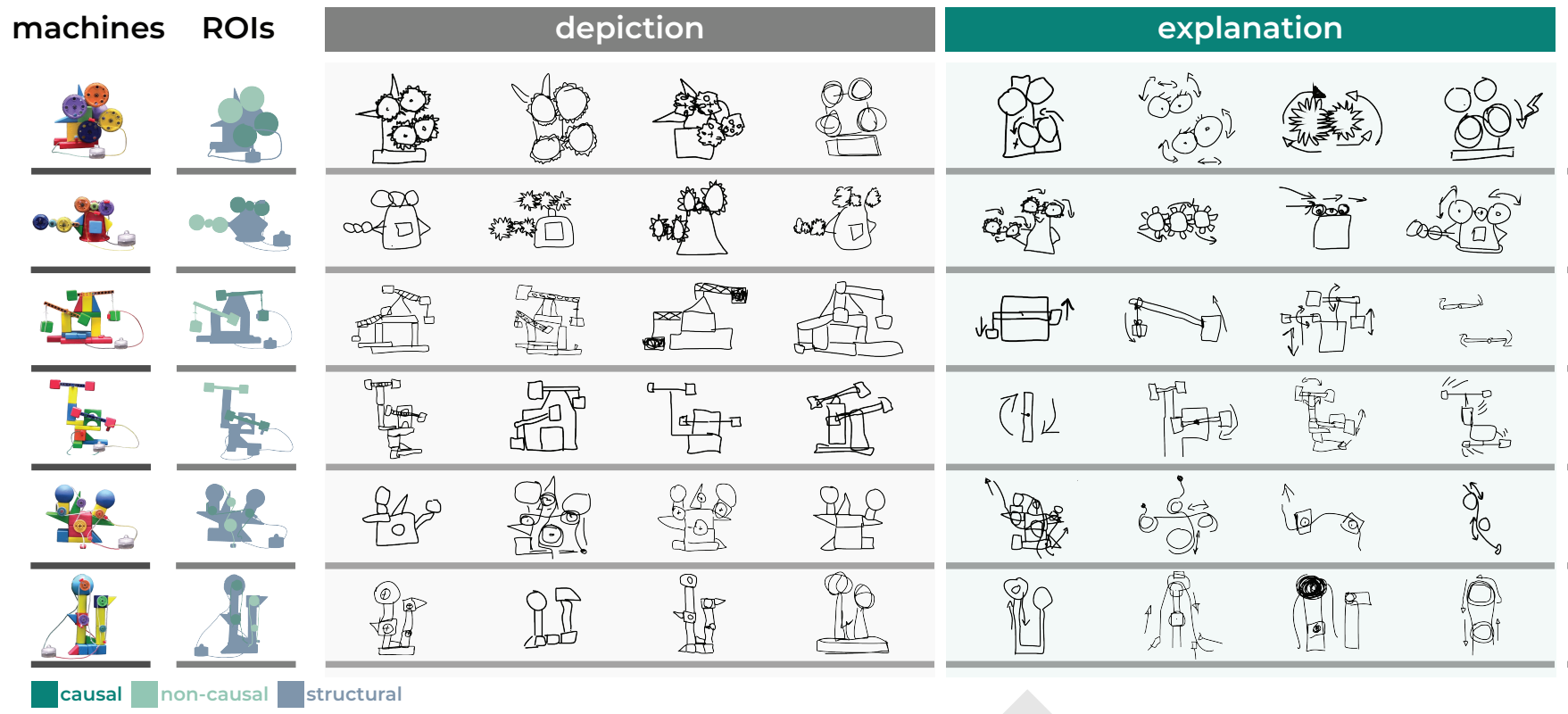

Fig. 2. Study 1: Visual Production Dataset. Left: Each machine consisted of multiple mechanical and structural elements. Each region-of-interest (ROI) image indicates the location of both causally relevant and non-causally relevant mechanical elements. Right: Example depictive and explanatory drawings.

These results suggest that the goal of communicating mechanistic knowledge leads people to produce drawings that place greater emphasis on causally relevant components and how they move, and less emphasis on static components, even if they are visually salient.

Beyond counting how many strokes were used to represent each part, we leveraged the inherently spatial information conveyed by each stroke to ask how well they preserved the size and relative locations of the parts they represented. One possibility is that explanatory drawings focus on causally relevant elements, but still faithfully preserve their visual properties. Alternatively, explanatory drawings may distort their visual properties, perhaps by making them appear larger or in a more prominent location. To distinguish these possibilities, we first grouped strokes from each drawing that were tagged with the same part label to define a spatial region within the drawing representing that part. We then computed the difference between the size and location of the drawn part and the size and location of the actual part as it appeared in the video demonstration (for additional details, please see Materials and Methods).

We found that mechanical elements were consistently drawn larger in explanations than in depictions (explanation: 72.4px, depiction: $60.8 \mathrm{px}, b=8.411, t=1.974, p=0.0496)$, in addition to being drawn somewhat further from their actual locations, relative to other parts of the machine (explanation: $75.09 \mathrm{px}$, depiction: $62.3 \mathrm{px}, b=11.615, t=3.153, p$ $=0.18 e-2)$. These findings are consistent with the notion that when explaining how a machine functions, people distort the appearance of functionally relevant parts to make them more salient and discount the importance of preserving exact spatial relationships. As a whole, Study 1 provides evidence that having the goal of communicating mechanistic knowledge systematically affects the kind of information people prioritize when producing visual explanations.
Study 2: How well do visual explanations support accurate inferences? To what extent do these communicative strategies actually impact the inferences made by naive observers relying only upon these explanations to learn about the objects they refer to? To answer this question, in Study 2 we recruited naive participants to view the drawings from Study $1 \mathrm{~A}$, and measured how well they could identify the original machine (Study 2A), localize the key causal element (Study 2B), and select the appropriate action (Study 2C) to turn on the light.

Study 2A: Object identification. The goal of Study $2 \mathrm{~A}$ was to test the hypothesis that reduced emphasis on structural elements in visual explanations would make it harder to match the drawing to the original machine, relative to depictions. To test this hypothesis, a group of naive participants $(\mathrm{N}=52)$ completed a visual search task. On each trial, participants were presented with randomly sampled drawing from Study $1 \mathrm{~A}$, surrounded by a circular array containing color photos of each of the six machines. Their goal was identify which machine the drawing represented by clicking the corresponding photo quickly and accurately (Fig. 4A). We found that participants were reliably above chance performance for both types of drawings (explanation: $b=0.561, z=3.94, p=$ 8.16e -5 ; depiction: $b=1.28, z=10.11, p=2 e-16$; Fig $4 \mathrm{~A})$. Consistent with our primary hypothesis, we found that participants were both slower (correct trials only: explanation: 2646ms; depiction: 2339ms; $b=1.019 e-1, t=3.26, p=$ $1.29 e-3$; Fig. $4 \mathrm{~A}$ ) and less accurate when cued with a visual explanation than with a depiction (explanation: $62.4 \%$; depiction: $76.7 \% ; b=-0.899, z=-2.89, p=3.75 e-3$; Fig. $4 \mathrm{~B}$, left). These results suggest that our manipulation of communicative goals in Study 1A measurably impacted how well viewers could extract relevant information from each type of drawing, such that depictive drawings were more informative about the identity of the target machine. 
A

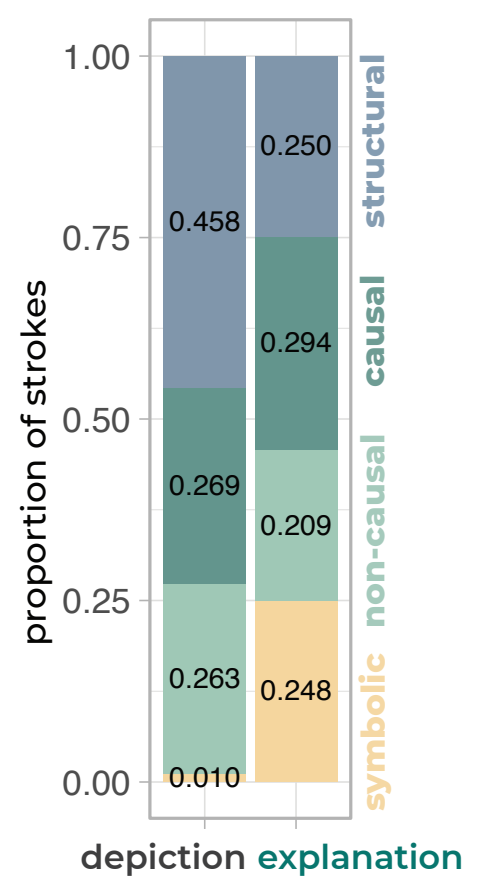

B
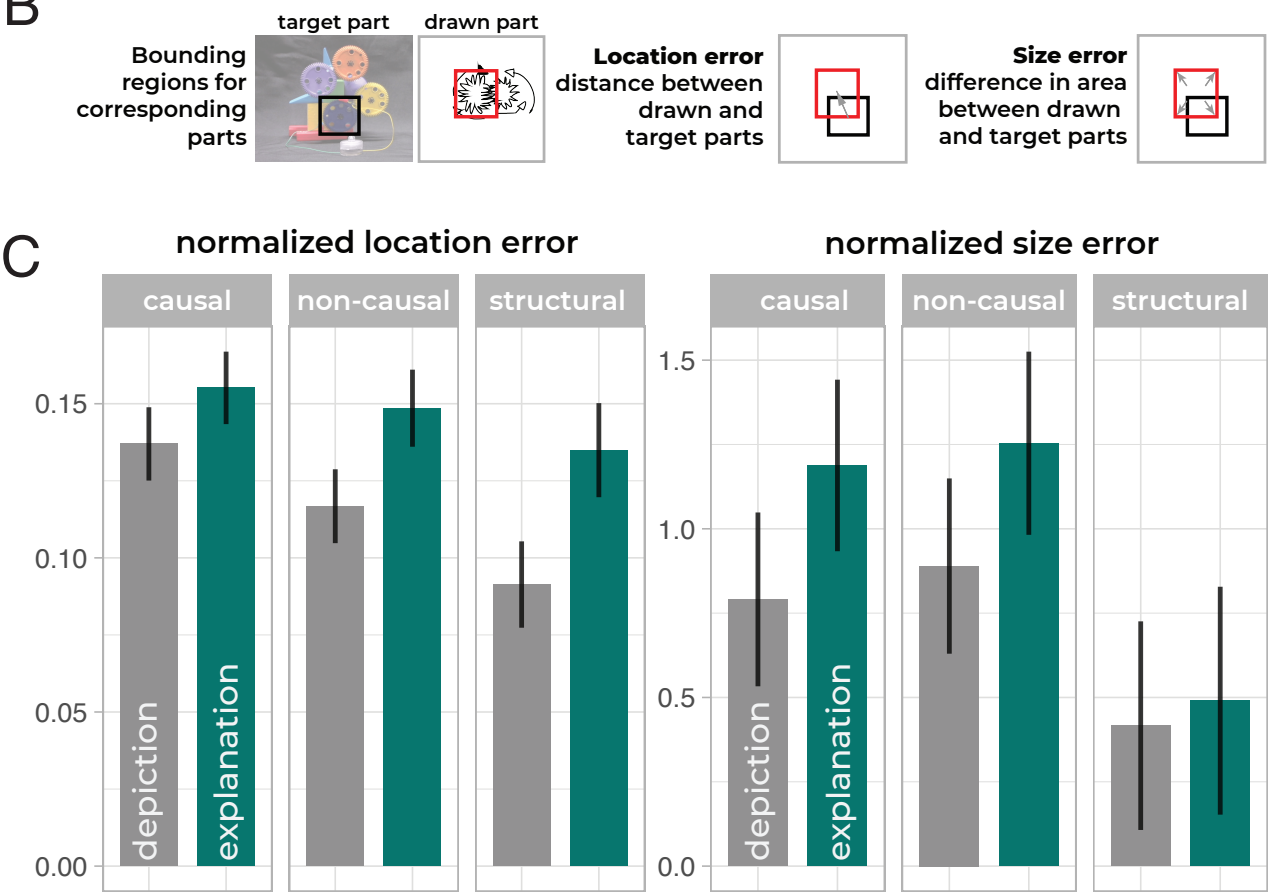

Fig. 3. Study 1: Results. A: Proportion of strokes conveying different semantic information: causal strokes representing mechanical elements that turned the light on; non-causal strokes representing mechanical elements that did not; structural strokes representing static elements; and symbolic strokes, including arrows and other marks indicating motion and interactions between elements. B: Accuracy of spatial information in drawings was estimated by defining bounding regions for corresponding parts in each drawing and video, then computing the difference in size and location between the drawn and target parts. C: Normalized location and size errors for different semantic part categories. Normalized location errors reflect relative differences between the target and drawn parts, rescaled by the size of the object. Normalized size errors reflect relative differences between the target and drawn parts, rescaled by the size of the target part. Error bars represent $95 \%$ Cls.

Study 2B: Causal part identification. An effective visual explanation not only highlights causally relevant information, but also helps viewers connect this information back to the elements they correspond to in the target system. The goal of Study 2B was to test the hypothesis that the greater emphasis that explanatory drawings placed upon causally relevant parts of the machine would indeed make it easier for viewers to locate those parts. To test this hypothesis, each participant in a new sample $(\mathrm{N}=305)$ was cued with a randomly sampled drawing of each of the six machines, with explanatory and depictive drawings appearing with equal probability. They were then shown a color photo of the machine with two mechanical elements highlighted and asked to indicate which element could be used to turn on the light. One of these mechanical elements was causal and the other was non-causal, such that any bias towards selecting the causal element would reflect the degree to which a drawing facilitated perceptual discrimination of the most functionally important parts of the machine.

We found that both types of drawings supported abovechance performance (explanation: $b=0.849, t=10.53, p$ $2 e-16$; depiction: $b=0.919, z=13.04, p 2 e-16$; Fig. $4 \mathrm{~B})$, suggesting that both types of drawings carried meaningful signal about the identity of the causally relevant parts. However, we did not find evidence for an advantage for explanatory drawings: on correct trials, participants took a similar amount of time to make their response (explanation: $3488 \mathrm{~ms}$; depiction: $3424 \mathrm{~ms}, b=-1.971 e-2, t=-0.287, p=0.775$; Fig. 4B). If anything, participants were less accurate when cued with a visual explanation than with a depiction (explanation: $68.5 \%$; depiction: $72.3 \%, b=-0.516, z=-2.601, p=0.929 e-2$; Fig. 4B). Taken together, these results show that the the greater visual emphasis on causal elements in explanatory drawings did not necessarily translate to improved ability to identify these parts in situ. Rather, they suggest that there may be more to the construction of an effective visual explanation than displaying the most functionally important entities more prominently.

Study 2C: Causal action selection. In addition to conveying which parts of the machine are most causally relevant, an effective visual explanation also helps a viewer infer what kind of intervention is needed to produce the desired effect. The goal of Study $2 \mathrm{C}$ was to test the possibility that explanatory drawings may outperform depictions in helping viewers infer the appropriate action to perform when attempting to turn on the light, perhaps owing to the presence of arrows and motion lines in these drawings.

Each participant in this new sample $(\mathrm{N}=270)$ was presented with a randomly sampled drawing of each of the six machines, and then asked to select the action that they thought they needed to perform in order to turn on the light. For each machine, participants selected from among three options: Pull, Push, and Rotate (Fig. 4C). Surprisingly, neither visual explanations nor depictions supported performance above chance (chance $=33 \%$; explanation: $b=-0.29, z=-2.98, p=$ $2.87 e-3$; depiction: $b=-1.023, z=-9.72, p=2 e-16$; Fig. $4 \mathrm{C}$ ). We found that participants more accurately identified the correct action when cued with a visual explanation 
A

object identification

Which object does

the drawing correspond to?
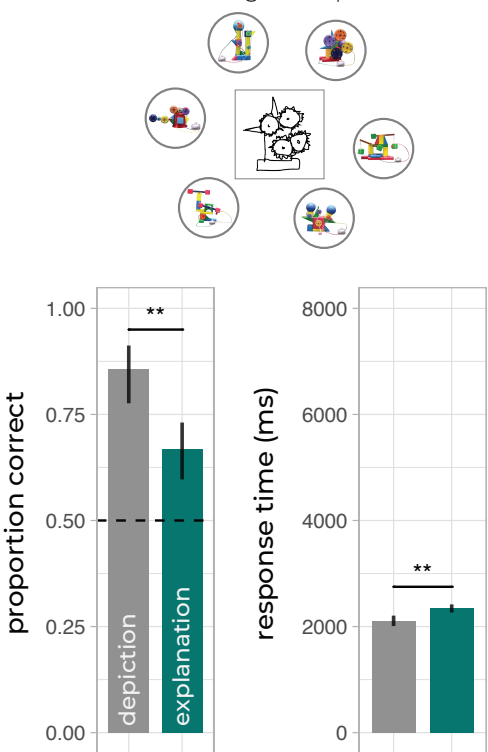

\section{B}

Which part of the object should be moved to turn on the light, based on the drawing?
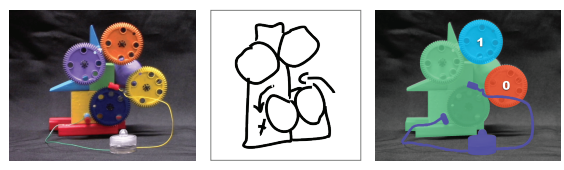

- - - study (3s) - - - - -study (3s) - - - - -press 1 or O-

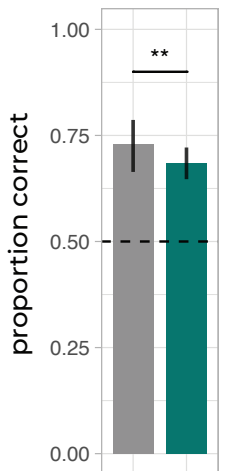

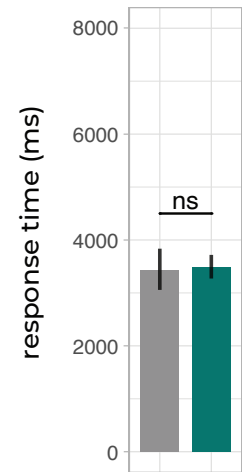

C causal action selection

Which action is needed to operate the object, based on the drawing?

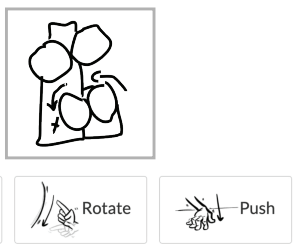

Fig. 4. Study 2: Visual Inference Tasks and Results. A: In Study 2A, participants identified the machine that matched each drawing. B: In Study $2 \mathrm{~B}$, participants identified which part of the machine they should intervene on to turned on the light bulb. $C$ : In Study 2C, participants inferred which action they would need to perform to turn on the light. Error bars represent $95 \%$ Cls.

(explanation: 41.5\%; depiction: $26.8 \%, b=0.662, z=3.72$, $p=2.01 e-4$; Fig. 4C). Between conditions, they took a similar amount of time to make their response (correct trials only, explanation: 6085ms; depiction: $4975 \mathrm{~ms}, b=0.0887, t$ $=0.691, p=0.4904$; Fig. $4 \mathrm{C})$, suggesting that the greater accuracy was unlikely to be due to a speed-accuracy tradeoff. Taken together with the results of Study 2B, these findings suggest that some aspect of explanatory drawings supported naive viewers' ability to figure out which action was needed to interact with the machine, even if they did not help them identify which part of the machine to interact with.

\section{Discussion}

Explanatory visualizations are a crucial tool for transmitting mechanistic knowledge, a key goal of science communication and STEM education. Nevertheless, we lack a detailed understanding of what ordinary people think is relevant when trying to explain how something works, as well as how these visual explanations guide people towards appropriate inferences. Towards closing this gap, we investigated what information people prioritize when drawing visual explanations of simple mechanical objects, and measured how well these explanations enabled other people to learn about these objects based on their drawings alone. We found that people spontaneously emphasized functionally important parts of these objects when producing an explanation, using more strokes to draw these parts and making them appear larger than when they only aimed to produce a visually accurate drawing of the object. They also drew abstract symbols, including arrows and motion lines, suggesting that they believe that providing an explanation means going beyond drawing physical components of the same object. While these explanatory drawings succeeded in communicating to other people which action was needed to interact with the object, they did not necessarily make it easier to connect key parts in the drawing to key parts of the object itself. Taken together, our findings show that ordinary people display sensitivity to information about functional organization (i.e., how parts move and interact) when asked to produce a visual explanation, in a way that systematically trades off with information about structural organization (i.e., what parts look like and where they are).

Our findings contribute to a growing body of work characterizing explanatory preferences (30) and the cognitive consequences of producing explanations $(19,28,29,32,33)$. In particular, participants who performed our visual explanation task prioritized causally relevant information at the expense of other salient information. This tendency is broadly consistent with previous evidence that individuals who are prompted to produce verbal explanations tend to privilege more abstract, functional properties over salient, perceptual features $(28,29)$. However, rather than focusing on what is learned by the individuals producing the explanations, our studies examined how the balance of structural and functional information in visual explanations guide inferences made by downstream learners. We found that the explanatory drawings elicited in our study supported certain inferences to a greater degree than others, suggesting that explanations are not necessarily superior to depictions in all settings, but rather a specific tool for conveying knowledge cast at a particular level of abstraction.

Future work should ask how different kinds of explanatory goals impact what information people believe to be important to include in their explanations. In our study, participants were cued to produce drawings explaining how the machines functioned to produce the desired effect. However, based on these instructions, drawers may have inferred one of two valid explanatory goals: first, to explain the specific mechanisms 
that cause the desired effect for this machine (i.e, how these gears turn the light on); and second, to explain the general principles governing the class of mechanisms used by the machine (i.e., how gears work in general). If some participants followed the latter interpretation, they might have produced visual explanations pitched at a higher level of abstraction, perhaps accounting for the lack of advantage in communicating the identity of the causally relevant part relative to depictions Future studies could tease apart these general and specific explanatory goals to understand to what extent the tradeoff between functional and structural information depends on how general a visual explanation is intended to be.

Another key direction for future work is to examine how expertise influences visual explanation behavior. The participants in our studies were unlikely to have received specific training in how to design effective visual explanations, and thus it may not be surprising that the explanations they produced did not outperform depictions in supporting identification of causally relevant parts. One potential explanation for this finding is that, by frequently omitting other (non-causal) mechanical parts and structural parts, these explanations failed to provide enough contextual information to help viewers situate the causally relevant part relative to the rest of the object. Future work could test this hypothesis by prompting drawers to take the perspective of a naive viewer (34), to examine whether they would be more likely to include enough additional structural information to produce more informative visual explanations. Such evaluations may help to clarify the role of social reasoning and pedagogical expertise in the production of effective explanations.

Overall, advancing our understanding of how visual explanations communicate mechanistic knowledge may have broad implications for psychological theories of explanatory behavior as well as for the role of visualizations in scientific learning and pedagogy.

\section{Materials and Methods}

Materials and data availability All experimental materials, study preregistrations, data, and analysis code are publicly available in our GitHub repository: https://github.com/cogtoolslab/causaldraw_ public2021.

\section{Study 1A: Production of visual explanations and depictions}

Participants. In this and all subsequent experiments, participants provided informed consent in accordance with the UC San Diego IRB. 124 participants (33 male; mean age $=21.1$ years) were recruited from the UC San Diego study pool for the stimulus validation experiment. Data from 6 sessions were excluded for technical problems (i.e., videos did not load). 52 participants (29 male; mean age $=39.1$ years) were recruited from Amazon Mechanical Turk for the drawing experiment. We excluded data from two participants who did not meet our predefined exclusion criteria for generating drawings that represented the target stimuli.

Stimuli. We designed six novel machines composed from simple mechanical elements (i.e., gears, levers, pulleys). There were two machines employing each type of element. Half of the mechanical elements in each machine were causal, meaning they could be used to produce a desired effect (i.e., turn on a light); the other half of mechanical elements were non-causal. To match how visually salient they were, the causal and non-causal elements within a machine were always of the same type (e.g., gear), and were approximately matched in size and number (Fig. 2).
Stimulus validation task procedure. To validate that the video demonstrations clearly conveyed how each machine worked, one group of participants was asked to identify which part of the machines they should intervene on to turn on the light. Specifically, after each video finished playing, participants were presented with color photographs of the machine, in which each mechanical part was color coded and given a unique numerical identifier (e.g., ' 1 ', '2', etc.). Participants then pressed the number key indicating which of the highlighted parts they thought they should intervene on to turn on the light, and to do so as quickly and as accurately as possible. At the beginning of the session, participants also completed a series of practice trials to gain familiarity with the task interface.

Visual production task procedure. Our second group of participants was presented with a series of 30 s videos demonstrating how to operate different novel machines in order to turn on a light bulb, and then was asked to produce drawings of them. Within each video demonstration, the demonstrator interacted with both the causal and non-causal mechanical elements twice each, in a counterbalanced order, to show that the causal element reliably turned on the light, whereas the non-causal element did not. After the video finished playing, participants were cued to produce one of two kinds of drawings of the machine: on explanation trials, they were prompted to produce visual explanations to help a naive viewer learn how the machine worked; on baseline depiction trials, they were prompted to produce visual depictions to help a naive viewer identify the machine by its appearance (Fig. 1). All participants produced three explanations and three depictions, in a randomized sequence, such that they drew one of each type of drawing for each type of machine. Participants used their cursor to draw in black ink on a digital canvas embedded in their web browser (canvas $=500 \mathrm{x}$ $500 \mathrm{px}$; stroke width $=5 \mathrm{px}$ ). Each stroke was rendered in real time on the participant's screen as they drew and could not be deleted once drawn. Participants were not limited in amount of time that they could spend drawing in each trial. At the beginning of each session, participants also completed two practice trials to familiarize them with the drawing interface.

Study 1B: Characterizing semantic content in visual explanations and depictions

Participants. 280 participants (238 male; $\mathrm{M}_{\text {age }}=38.9$ years) were recruited from from Amazon Mechanical Turk to provide semantic annotations of the drawings produced in Study 1. We excluded data from 28 participants, who did not meet our preregistered inclusion criteria (i.e., low accuracy on attention-check trials, response time $<5$ s).

Procedure. Annotators were presented with a set of 10 drawings that were randomly sampled from those drawn in the visual production experiment, as well as reference color photographs of the original machines. The photographs were color-coded and numbered by different parts (e.g., gear, light, structural) to help participants segment and identify them. Annotators were asked to tag each individual stroke based on what they interpreted it to represent. To facilitate consistent labeling, they were provided with a menu of part labels specific to each machine (e.g., gear, light, structural). If a stroke depicted a symbol (e.g., arrow, motion line), annotators were asked to additionally label which element(s) the symbol referred to. If a stroke's referent was not clear, annotators were able to select an "I don't know" option.

Preprocessing annotation data. We found that $64.9 \%$ of strokes received the same label by all three annotators, and $95.0 \%$ of strokes received the same label by at least two of the three annotators. $5.0 \%$ of strokes did not reach a majority consensus and received more annotations to resolve this conflict. Moreover, within visual explanations, $55.5 \%$ of strokes received the same label by all three annotators, and $93.2 \%$ of strokes received the same label by at least two of the three annotators. Within depictions, $75.0 \%$ of strokes in depictions received the same label by all three annotators, and $96.9 \%$ of strokes received the same label by at least two of the three annotators. In subsequent analyses, we collapsed over interannotator variation and assigned the modal label to strokes which had been given the same label by at least two annotators. For the remaining strokes that did not receive a modal label, we randomly sampled 
476 an annotation from the set of annotations that had been assigned 477 to it. We also excluded 5 drawings from subsequent analyses that 478 were deemed to be entirely uninterpretable.

Spatial error analysis. To measure how accurately the drawings from Study $1 \mathrm{~A}$ preserved information about the spatial properties of each part of the target object, we used the following procedure to quantify the deviation between the the relative sizes and locations of drawn parts and the sizes and locations of the target parts they represented, as they appeared in still frames taken from the video demonstrations.

First, to compute the size and location of drawn parts, we grouped all strokes within a drawing that were tagged with the same semantic label, then determined the coordinates of the rectangular bounding box containing those parts. For example, if a drawing contained strokes representing four different gears and some structural elements, then this step would yield five bounding boxes, one for each gear, and the fifth containing all structural elements. Strokes representing symbols and/or the light bulb were excluded from analysis.

Next, to compute the size and location of target parts, we color-coded each part of the still images of the machines in Adobe Photoshop and grouped all the pixels of the same color. We then calculated the coordinates of the individual bounding boxes for each part. Because the goal of our analysis was to measure how accurately drawings preserved relative size and location information, we aligned each drawing to its target object before computing size and location errors. Specifically, we calculated the overall bounding box containing all strokes and the overall bounding box of the target object containing all parts and then applied the translation and scaling transformations needed to align the two.

To calculate raw location error for a given part, we computed the euclidean distance between the centroid of the bounding box for each drawn part and the centroid of the bounding box for the target part. The raw location error for the drawing as a whole was computed by taking the mean of these distances across all parts that appeared in the drawing. We then divided this raw location error by the length of the diagonal of the object's bounding box to derive a normalized measure of location error, enabling more straightforward aggregation of location error estimates between objects of different sizes.

To calculate raw size error for a given part, we calculated the difference in area between the bounding box of the drawn part and the bounding box for the target part. We then normalized this raw size error by dividing it by the area of the target part, such that values larger than 1 indicated that the part appeared larger in the drawing than its referent in the still image, and values smaller than 1 indicated that the part appeared smaller in the drawing than its referent. The normalized size error for a drawing as a whole was computed by taking the mean across all parts that appeared in the drawing.

\section{Study 2A: Object identification.}

Participants. 52 participants ( 24 male; $\mathrm{M}_{\text {age }}=20.5$ years $)$ were recruited from the UC San Diego study pool. Data from two sessions were excluded for technical problems (i.e., inability to click on images).

Procedure. The goal of Study 2A was to measure how well drawings conveyed information about the identity of the target machine. Participants completed a visual search task in which each participant was presented with all 300 drawings from Study $1 \mathrm{~A}$ in a randomized sequence. At the beginning of each trial, participants moved their cursor to a crosshair displayed at the center of an empty display. When ready, participants clicked this crosshair to reveal a single drawing ( $175 \times 175 \mathrm{px})$ at that location, surrounded by a circular array of six color photographs (125px x $100 \mathrm{px}$, radius $=250 \mathrm{px}$ ), one of each machine (Fig. 4A). The angular distance between each photo was constant (i.e., 60 degrees), and the locations of the machine photos were randomized between trials. Participants were instructed to click on the machine that the drawing corresponded to as quickly and accurately as possible. At the beginning of the session, participants completed 6 practice trials where they were cued with photos of each machine (instead of drawings), and had to click on the matching photo in the array.
Statistical analyses. To investigate how well these drawings support participants' ability to identify the machines above chance, we fit a null model predicting identification accuracy that included random intercepts for different production participants. Although there were 6 machines, we defined chance-level performance at $50 \%$, a theoretical upper bound reflecting our expectation that confusions would be most likely to arise between machines of the same type (e.g., gears).

To evaluate our hypothesis that participants would be slower for explanations than depictions, we fit a linear mixed-effects model predicting response time from condition, as well as additional predictors controlling for the number of each type of stroke within a drawing (i.e., causal, non-causal, structural, symbol), the interaction between condition and the number of each type of stroke, and random intercepts for individual drawings and participant.

To evaluate our hypothesis that participants would be less accurate for explanations than depictions, we fit a mixed-effects logistic regression model to predict individual trial outcomes, with the same fixed and random effects structure as our response-time model above.

Study 2B: Causal part identification.

Participants. 305 participants (100 male; $\mathrm{M}_{\mathrm{age}}=28.4$ years) were recruited from Prolific $(\mathrm{N}=99)$ and the study participant pool at UCSD $(\mathrm{N}=206)$. 8 data sessions were excluded for technical problems with displaying the experimental stimuli (e.g., the videos did not load).

Procedure. The goal of Study 2B was to measure how well drawings conveyed information about which part was most causally relevant for operating each machine. Participants were presented with a randomly sampled set of 6 drawings from Study 1A, one of each machine, in a randomized sequence. On every trial, participants were presented with three images laid out in a horizontal array, appearing in succession: first, a color photograph of one of the machines appeared on the left; second, after a 3s delay, a drawing of it appeared in the middle; and third, after another 3s delay, another photograph of the same machine appeared on the right, this time with one causal element and one non-causal element highlighted in different colors (Fig. 4B). Participants were instructed to press a key (i.e., either 0 or 1 ) to indicate which of the highlighted elements they would intervene on to turn on the light, and to do so as quickly and accurately as possible. At the beginning of the session, participants completed a series of practice trials in which they were familiarized with the task interface.

Statistical analyses. As in Study 2A, we fit a null model predicting identification accuracy that included random intercepts for different production participants to evaluate the degree to which participants performed above chance.

To evaluate whether participants would be faster in identifying the causal part when presented with a visual explanation rather than a depiction, we constructed a linear mixed-effects model to predict response time from condition, as well as additional predictors controlling for the number of each type of stroke within a drawing (i.e., causal, non-causal, structural, symbol) and the interaction between condition and the number of each type of stroke, and random intercepts for individual drawings and participant.

To evaluate our hypothesis that participants would be more accurate for explanations than depictions, we fit responses with the same statistical model as in Study 2A.

\section{Study 2C: Causal action selection.}

Participants. 270 participants $\left(75\right.$ male; $\mathrm{M}_{\text {age }}=21.3$ years $)$ were recruited from the UC San Diego study pool. Data from three sessions were excluded for technical problems (i.e., videos did not load).

Procedure. The goal of Study $2 \mathrm{C}$ was to measure how well drawings conveyed information about the type of action needed to operate each machine. Participants were presented with a random set of 6 drawings from Study 1A, one of each machine, in randomized sequence. On each trial, participants saw a single drawing, under which there were 3 buttons labeled as "Pull", "Push", "Rotate" 
and "I don't know" (Fig. 4C). Participants were instructed to click the button that corresponded to the action needed to operate the machine, based on their interpretation of the drawing, and were told to prioritize accuracy. At the beginning on of the session, participants completed a series of practice trials in which they were familiarized with the task interface.

Statistical analyses. To evaluate the degree to which participants performed the task above chance, we fit a null model predicting accurate responses that was identical in structure to that used in Study $2 \mathrm{~A}$ and $2 \mathrm{~B}$.

To evaluate differences in how quickly participants could identify the correct action, we fit a linear mixed-effects model predicting response time from condition, as well as additional predictors controlling for the number of each type of stroke within a drawing (i.e., causal, non-causal, structural, symbolic), the interaction between condition and the number of each type of stroke, and random intercepts for individual drawings and participants.

To evaluate differences in how accurately participants could identify the correct action, we fit their responses using the same type of statistical model as in Study 2A and 2B, with two simplifying modifications: because visual explanations consistently contained more strokes illustrating symbols than depictions, we removed the predictor for number of strokes corresponding to symbols, as well as the interaction between condition.

ACKNOWLEDGMENTS. Thanks to the members of the Cognitive Tools Lab and Early Learning \& Cognition Lab at University of California, San Diego for helpful discussion. This work was supported by an NSF CAREER Award \#2047191 to J.E.F. A subset of these findings were presented as part of the Proceedings of the 43rd Annual Meeting of the Cognitive Science Society.

1. DA Lagnado, S Sloman, The advantage of timely intervention. J. Exp. Psychol. Learn. Mem. Cogn. 30, 856 (2004)

2. M Steyvers, JB Tenenbaum, EJ Wagenmakers, B Blum, Inferring causal networks from observations and interventions. Cogn. science 27, 453-489 (2003).

3. R Boyd, PJ Richerson, J Henrich, The cultural niche: Why social learning is essential for human adaptation. Proc. Natl. Acad. Sci. 108, 10918-10925 (2011).

4. G Csibra, G Gergely, Natural pedagogy. Trends Cogn. Sci 13, 148-153 (2009).

5. FC Keil, KL Lockhart, Beyond cause: The development of clockwork cognition. Curr. Dir. Psychol. Sci. 30, 167-173 (2021).

6. W Bechtel, Mechanism and biological explanation. Philos. science 78, 533-557 (2011).

7. WC Wimsatt, Reductionism, levels of organization, and the mind-body problem in Consciousness and the brain. (Springer), pp. 205-267 (1976).

8. KH Corriveau, KE Kurkul, "why does rain fall?": Children prefer to learn from an informant who uses noncircular explanations. Child development 85, 1827-1835 (2014).

9. D Kelemen, E Rosset, The human function compunction: Teleological explanation in adults. Cognition 111, 138-143 (2009).

10. W Bechtel, Constructing a philosophy of science of cognitive science. Top. Cogn. Sci. 1, 548-569 (2009).

11. KL Lockhart, A Chuey, S Kerr, FC Keil, The privileged status of knowing mechanistic information: An early epistemic bias. Child development 90, 1772-1788 (2019).

12. A Chuey, $K$ Lockhart, $M$ Sheskin, $F$ Keil, Children and adults selectively generalize mechanistic knowledge. Cognition 199, 104231 (2020).

13. M Hegarty, The cognitive science of visual-spatial displays: Implications for design. Top. cognitive science 3, 446-474 (2011).

14. B Tversky, Prolegomenon to scientific visualizations in Visualization in science education. (Springer), pp. 29-42 (2005).

15. RE Mayer, Multimedia aids to problem-solving transfer. Int. J. Educ. Res. 31, 611-623 (1999).

16. M Scaife, Y Rogers, External cognition: how do graphical representations work? Int. journal human-computer studies 45, 185-213 (1996).

17. M Hegarty, MA Just, Constructing mental models of machines from text and diagrams. J. memory language 32, 717-742 (1993).

18. M Hegarty, S Kriz, C Cate, The roles of mental animations and external animations in understanding mechanical systems. Cogn. instruction 21, 209-249 (2003).

19. E Bobek, B Tversky, Creating visual explanations improves learning. Cogn. Res. Princ. Implic. 1, 27 (2016).

20. JE Fan, Drawing to learn: How producing graphical representations enhances scientific thinking. Transl. Issues Psychol. Sci. 1, 170 (2015).

21. JD Gobert, JJ Clement, Effects of student-generated diagrams versus student-generated summaries on conceptual understanding of causal and dynamic knowledge in plate tectonics. J. Res. Sci. Teaching: The Off. J. Natl. Assoc. for Res. Sci. Teach. 36, 39-53 (1999).

22. NS Newcombe, Seeing relationships: Using spatial thinking to teach science, mathematics, and social studies. Am. Educ. 37, 26 (2013).

23. RE Mayer, Systematic thinking fostered by illustrations in scientific text. J. educational psychology 81, 240 (1989).

24. AM Glenberg, WE Langston, Comprehension of illustrated text: Pictures help to build mental models. J. memory language 31, 129-151 (1992).

25. JH Larkin, HA Simon, Why a diagram is (sometimes) worth ten thousand words. Cogn. science 11, 65-100 (1987).
26. WS Cleveland, Graphs in scientific publications. The Am. Stat. 38, 261-269 (1984).

27. K Hentschel, Visual cultures in science and technology: A comparative history. (Oxford University Press, USA), (2014).

28. CH Legare, T Lombrozo, Selective effects of explanation on learning during early childhood J. Exp. Child Psychol. 126, 198-212 (2014).

29. CM Walker, T Lombrozo, CH Legare, A Gopnik, Explaining prompts children to privilege inductively rich properties. Cognition 133, 343-357 (2014).

30. T Lombrozo, Explanatory preferences shape learning and inference. Trends Cogn. Sci. 20 748-759 (2016).

31. MT Chi, KA VanLehn, The content of physics self-explanations. The J. Learn. Sci. 1, 69-105 (1991).

32. MT Chi, N De Leeuw, MH Chiu, C LaVancher, Eliciting self-explanations improves understanding. Cognitive Science 18, 439-477 (1994).

33. L Fiorella, Q Zhang, Drawing boundary conditions for learning by drawing. Educ. Psychol. Rev. 30, 1115-1137 (2018).

34. P Shafto, ND Goodman, TL Griffiths, A rational account of pedagogical reasoning: Teaching by, and learning from, examples. Cogn. Psychol 71, 55-89 (2014).

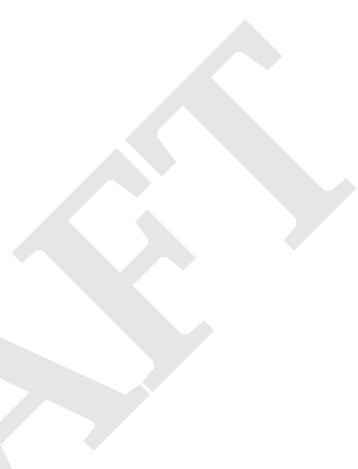

